\title{
Schulterluxationen, Indikation zur operativen Versorgung
}

\author{
Jens Richter, Andreas Dávid
}

\section{Zusammenfassung}

Mehr als 95\% der Schulterluxationen erfolgen nach ventral. Das Verletzungsmuster ist unterschiedlich, ein Labrumabriss zählt in mehr als 85\% dazu. Entscheidender sind die Prognose einer Rezidivluxation ist jedoch der zusätzliche Abriss des Lig. glenohumerale inferius und das Patientenalter. Ein Hill-Sachs-Defekt gewinnt erst ab einem Anteil von $30 \%$ an der Humeruskopfzirkumferenz an Bedeutung. Die Indikation zur sofortigen Operation ist die verhakte, nicht geschlossen zu reponierende Verrenkung (zumeist nach dorsal). Frühzeitig sollten relevante Pfannenrandfrakturen sowie Labrum-Kapselabrisse bei jungen Patienten unter 26 Jahren und schulteraktiven Sportlern fixiert werden. Während noch vor wenigen Jahren diese vorderen Schulterluxationen geschlossen reponiert und unabhängig vom Alter sowie vom Leistungsprofil des Patienten früh-funktionell nachbehandelt wurden, setzt mit Entwicklung arthroskopischer minimal invasiver Techniken ein Umdenken zur Differenzierung ein. Vor einem allgemeinen Einsatz der Schulterarthroskopie nach vorderer Luxation sollte gewarnt werden, da es sich letztendlich um einen operativen Eingriff mit möglichen Nebenwirkungen handelt. Für den modernen Behandlungsalgorithmus ist zunächst eine strenge Trennung zwischen traumatischen und atraumatischen Erstluxationen anzustreben. Während die erste Gruppe nach dem TUBS-Schema (traumatisch-unilateral-Bankartläsion-surgery) von einer arthroskopischen Technik deutlich profitieren kann, gilt für die zweite Gruppe $\mathrm{Zu}$ rückhaltung entsprechend dem sog. AMBRII-Schema (atraumatisch-multidirektional-bilateral-Rehabilitation vor inferiorem Kapselshift und Intervallverschluss). Anatomische artikularseitige Rekonstruktionen mit einer Refixation des Limbus-Kapselkomplexes nach Bankart werden heute als Standard bevorzugt, auch bei Rezidiven. Extraartikuläre Eingriffe sind individuellen Indikationen vorbehalten. Arthroskopische Verfahren erfordern ein aufwändiges und genau auf die Fixationstechnik abgestimmtes Equipment. Mit differenzierter Indikationsstellung, Patientenselektion und zunehmender Lernkurve gelingt es in aktuellen Studien die Erfolgsrate dem des offenen Bankartrepair anzunähern.

\section{Einleitung}

Die Schulterluxation ist durch einen kompletten Kontaktverlust zwischen Oberarmkopf und Gelenkpfanne definiert. $\mathrm{Zu}$ etwa 95\% verrenkt der Humeruskopf nach ventral und $\mathrm{zu} 3 \%$ nach dorsal (Tab.1). Die Luxatio erecta bezeichnet

OP-JOURNAL 2002; 18: 208-217

(C) Georg Thieme Verlag Stuttgart - New York einen Sonderfall, bei dem der weit abduzierte Arm durch Anschlag des Humerushalses gegen das Akromion den Kopf nach kaudal aus der Pfanne drückt.

Bei einer Inzidenz von 17 Fällen auf 100000 Einwohner [15], entsprechend einer dänischen Studie, würde dies für die Bundesrepublik Deutschland (80 $\times 10^{6}$ Einwohner) etwa 13600 primäre Schulterluxationen pro Jahr bedeuten. Dabei sind sportlich aktive Männer zwischen dem 20. und 40. Lebensjahr bevor- zugt betroffen [5,11]. Die Ursachen der Schulterverrenkung (Tab.1) kann man in traumatische (direkte - z.B. Sturz oder indirekte Krafteinwirkung - z.B. beim Kontaktsport wird der abgespreizte und angehobene Arm nach hinten außen gerissen) sowie nicht-traumatische Erstluxationen unterscheiden. Die hintere Luxation wird nicht selten durch direkte Krafteinwirkung (z.B. beim Kampfsport) oder durch Muskelkonvulsionen infolge eines epileptischen Anfalles bzw. einer Stromschlagverletzung (dabei gelegentlich auch beidseitig) verursacht. Zweitoder Rezidivluxationen werden in „unwillkürliche“ und „willkürliche“ (vom Patienten reproduzierbar) unterteilt. Die aus der Schulterluxation resultierende mögliche Instabilität kann unidirektional oder multidirektional sein. Koexistenzen mit einer vorbestehenden Hyperlaxizität der Schulter oder anderer Gelenke sind nicht selten.

\section{Erstbehandlung}

Luxationen des Schultergelenkes sind eine Notfallindikation zur sofortigen Reposition.

Zuvor sollte jedoch der neuro-vaskuläre Status im Hinblick auf mögliche Schäden des N. axillaris (Sensibilität im Bereich der Schulterkappe) sowie Läsionen des Armplexus (Fingerbeweglichkeit und Missempfindungen) dokumentiert werden. Eine vorhergehende Röntgendokumentation belegt zum einen die Luxationsrichtung, die wichtig ist für das anschließende Repositionsmanöver, zum anderen sollten hierdurch Therapieund mögliche rechtsrelevante Fragen (z.B. wie kam es zur Humeruskopffraktur?) geklärt werden. Einrenkungen bei vorderer Luxation des Schultergelenkes führen wir i.d.R. unblutig bzw. geschlossen in der Technik nach Hippokrates (Arzt zieht am Arm des liegenden Patienten gegen ein Widerlager in der Achsel) oder nach Arlt (Gewicht von mehren Kg zieht 
Tab. 1 Ursachen der Schulterluxation

\begin{tabular}{|c|c|c|c|}
\hline Luxationsrichtung & Ursache & & Anmerkung \\
\hline $\begin{array}{l}\text { anterior: } \\
\text { subkorakoidal oder } \\
\text { subglenoidal } \\
\sim 95 \%\end{array}$ & $\begin{array}{l}\text { traumatisch } \\
\text { atraumatisch }\end{array}$ & $\begin{array}{l}\text { - direkte Krafteinwirkung } \\
\text { - indirekte Krafteinwirkung } \\
\text { - allg. Kapsellaxizität } \\
\text { - repetitive Mikrotraumen } \\
\text { - Pfannendysplasie } \\
\text { - Humerustorsion } \\
\text { - Muskeldysbalance } \\
\text { - Rotatorenmanschettenruptur } \\
\quad \text { (Subskapularissehne) }\end{array}$ & $\begin{array}{l}\text { zumeist unidirektionale Instabilität } \\
\text { oft multidirektionale Instabilität }\end{array}$ \\
\hline selten: subklavikular & \multicolumn{3}{|c|}{ Rarität, zumeist durch schweres direktes Trauma } \\
\hline Luxatio erecta & \multicolumn{2}{|c|}{ Rarität, oft traumatisch - durch Hyperabduktion } & $\begin{array}{l}\text { oft kombiniert mit Tub.-majus-Fraktur und } \\
\text { Nervenverletzungen, erhöhte Reluxationsrate }\end{array}$ \\
\hline zentral: intrathorakal & \multicolumn{2}{|c|}{ Rarität, zumeist durch schweres direktes Trauma } & Begleitfrakturen, Thoraxtrauma \\
\hline superior: subakromial ${ }^{14}$ & \multicolumn{2}{|c|}{ Rarität, zumeist durch schweres direktes Trauma } & Begleitfrakturen \\
\hline posterior $^{9}<3 \%$ & traumatisch & $\begin{array}{l}\text { - durch epileptischen Anfall oder Stromunfall, } \\
\text { selten durch direktes Anpralltrauma }\end{array}$ & $\begin{array}{l}\text { wird häufig in Röntgenaufnahmen übersehen, } \\
\text { klinisch ist die Außenrotation eingeschränkt }\end{array}$ \\
\hline & atraumatisch & $\begin{array}{l}\text { - allg. Kapsellaxizität } \\
\text { - Pfannendysplasie } \\
\text { - neuromuskuläre Störungen }\end{array}$ & \\
\hline
\end{tabular}

am Arm des sitzenden Patienten, Widerlager bildet z.B. eine hohe Stuhllehne oder eine spezielle Rohrkonstruktion, wie sie auch für Extensionen verwendet wird). Dabei ist auf eine ausreichende Analgesie (z.B. auch intraartikulär) zu achten, um das Repositionsmanöver so schonend wie möglich durchzuführen. Verhakte Luxationen sind oftmals nur unter Kurznarkose zu reponieren und bei zusätzlicher Fraktur/Impression des Humeruskopfes ist auf dem Operationstisch ein offenes Vorgehen erforderlich.

Nach der Reposition werden die Weichen für die Weiterbehandlung gestellt. Die Dokumentation der Gelenkkongruenz hat auf Röntgenaufnahmen in 2 Ebenen (z. B. „true ap“ und axial) zu erfolgen. Mit einer Sonographie der Rotatorenmanschette gelingt der Ausschluss eines kompletten Sehnenabrisses in über 90\% [8]. Eine einfache Bandage mit angelegtem Arm (z. B. nach Gilchrist) wirkt nach Repositionen vorderer Luxationen schmerzlindernd und entspannt die vordere Kapsel. Dagegen empfiehlt sich zur Lagerung nach Reposition hinterer Schulterverrenkungen eine Spezialschiene, die den angelegten Arm in neutraler oder geringer Außenrotation halten. Während vor wenigen Jahren die meisten Patienten nach der Reposition mit einer Empfehlung zur konservativen Therapie ambulant behandelt wurden, rückt heute immer mehr das Interesse für die Folgeschäden an der Schulterkapsel und Möglich- keiten der frühzeitigen operativen Behandlung in den Vordergrund.

Bei der vorderen Schulterverrenkung wird der Oberarmkopf über den vorderen Pfannenrand und dem hier anheftenden Labrum hinausgehebelt. Dadurch gerät der dorso-kraniale Humeruskopf in die Zange zwischen Kapsel ventral und dem anterioren Pfannenrand dorsal, es entsteht die sog. „Hill-Sachs“-Delle, eine Labrumablösung mit oder ohne knöcherne Pfannenrandbeteiligung, Einrisse von Kapselbändern, Frakturen, Rotatorenmanschetten- oder Bizepssehnenrissen (Abb.1; Tab. 2).

\section{Rezidiv-Luxationen}

Aus retrospektiven Studien ist bekannt, dass das Risiko für weitere Verrenkungen vom Alter des Betreffenden abhängig ist. So beträgt dieses nach Untersuchungen von Rowe [23] für 10 - bis 20 -jährige $94 \%$, für 20 - bis 30 -Jährige $79 \%$ und für 30 - bis 40-Jährige Patienten $50 \%$.

Der Schwede L. Hovelius [11] bestätigte mit seiner Multizenterstudie die hohe altersbezogene Reluxationsrate von mehr als $50 \%$ bei Patienten, die zum Behandlungszeitpunkt jünger als 26 Jahre alt waren. Selbst eine 3- bis 4-wöchige Immobilisation konnte die hohe Rezidivrate nicht wesentlich beeinflussen. Lange Zeit wurden die Empfehlungen aus dieser Arbeit für ein früh-funktionelles Nachbehand- lungskonzept auch in der deutschsprachigen Literatur übernommen. Mit der Weiterentwicklung bildgebender und operativer Verfahren [Abb. 2] wuchs das Verständnis für die Rezidive induzierenden Verletzungen $[6,25]$ des Schultergelenkes (Tab.2). Nach Untersuchungen von Hintermann [10] liegen Bankart-Läsionen in $87 \%$, Kapselerweiterungen in $79 \%$ und eine Hill-Sachs-Delle in $68 \%$ sowie insuffiziente glenohumerale Bänder in 55\% der Luxationen vor. Einen Rotatorenmanschettenriss fand der Schweizer bei $14 \%$, eine hintere Labrumläsion in $12 \%$ sowie einen superioren Labrumriss vom SLAP-Typ bei 7\% seiner Patienten.

Die Laxizität, als vorbestehende Bindegewebskomponente des Kapsel-Bandapparates, wird im klinischen Alltag oftmals nur ungenügend für das operative Behandlungskonzept berücksichtigt.

Durch die klinische und intraoperative Untersuchung sollte zwischen einer unidirektionalen und einer multidirektionalen Instabilität sowie einer gleichzeitig bestehenden Hyperlaxizität der Schulter (die in $50 \%$ mit einer generellen Hyperlaxizität kombiniert ist) differenziert werden (Tab. 3).

Dazu werden das Sulkus-Zeichen, die vordere und hintere Schublade (Translation des Humeruskopfes) und das ventrale sowie dorsale „Apprehension“-Phänomen überprüft. Weiterhin sollte auch 


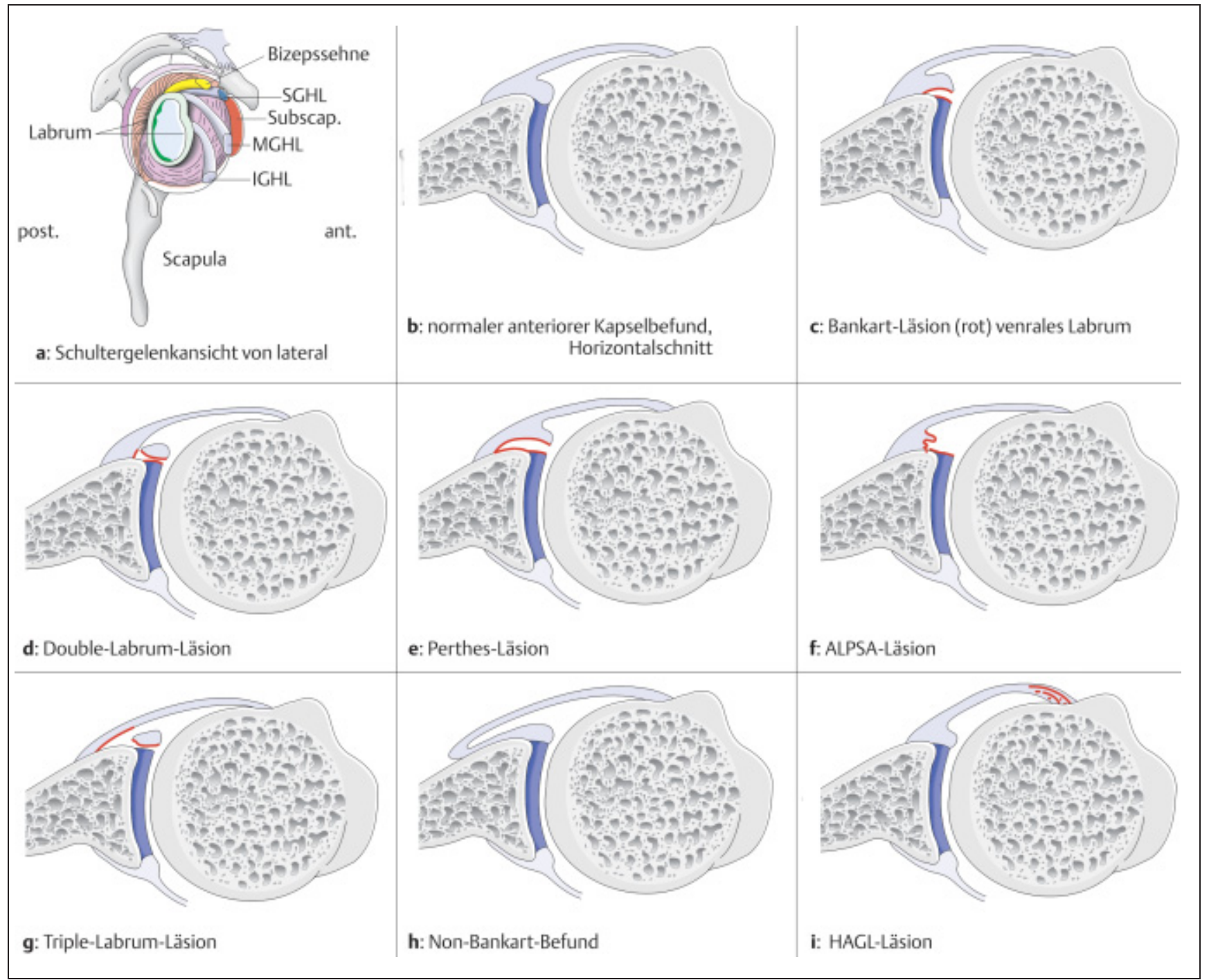

Abb.1a-i Labrum-Kapselläsionen im Rahmen der arthroskopischen Diagnostik.



Abb. 2 Double-Labrum-Läsion, Fäden (blau) zur Rekonstruktion schon eingebracht. nach Bandverletzungen an anderen Gelenken (z.B. Außenbänder, Kniebandläsionen, kontralaterale Schulter, Sportarten wie Gymnastik oder Schwimmen in der Vorgeschichte) gefragt werden. Patienten mit einem multidirektional instabilen Schultergelenk und zusätzlicher Hyperlaxizität haben die Erstluxation oftmals nach einem banalen Ereignis und leiden nicht selten an Subluxationen im Alltag (Ausdruck der ungenügenden Muskelkontrolle). Davon sind Patienten abzugrenzen, die ohne vorausgehendes Trauma eine willkürliche Luxation mit eigenständiger Einrenkung demonstrieren können. Fehlen hier die alltäglichen Subluxationsbeschwerden, so ist dies als positives Zeichen der erhaltenen Muskelkontrolle zu werten. Eine operative Behandlung sollte hier abgelehnt werden, da keine Arthroseentwicklung zu erwarten ist [24].

\section{Operative Behandlung der vorderen Schulterinstabilität}

Klassifikationen der Schulterinstabilität entstanden unter dem Gesichtspunkt, Ursachen und Therapiekonzepte rationell einander zuzuordnen. Verständlich und im klinischen Alltag gebräuchlich ist z.B. die Einteilung von Matsen [18].

Mit dem Begriff „TUBS“ wird der traumatischen unidirektionalen Instabilität durch Bankart-Läsion ein surgical repair zugeordnet. Dem stehen Instabilitäten vom „AMBRII“-Typ gegenüber. Hier erfolgt die Luxation atraumatisch, es liegt eine multidirektionale Instabilität vor, die oftmals auch bilateral auftritt. Die Therapie sollte konservativ sein mit Rehabilitation oder operativ mit einem inferioren Kapsel-shift und Intervallverschluss. 
Tab. 2 Labrum-Kapselverletzungen nach vorderer Schulterluxation

\begin{tabular}{|c|c|}
\hline Bankart-Läsion: & $\begin{array}{l}\text { Abriss des vorderen mittleren oder inferioren Labrums zwischen } 2 \text { und } 6 \text { Uhr* ohne Periosttasche, es ist kapselseitig noch } \\
\text { im Verbund. }\end{array}$ \\
\hline $\begin{array}{l}\text { Double-Labrum-Läsi- } \\
\text { on: }\end{array}$ & Das Labrum ist komplett sowohl vom Glenoid als auch von der Kapsel gelöst. \\
\hline $\begin{array}{l}\text { knöcherner Bankart- } \\
\text { Defekt: }\end{array}$ & Abscherfraktur als solides Fragment oder auch mehrfragmentär. \\
\hline Perthes-Läsion: & Labrum gemeinsam mit der Kapsel zwischen 2 und 6 Uhr vom Glenoidrand abgerissen, es bildet sich eine Tasche. \\
\hline ALPSA-Läsion: & Labrum mit Kapsel sind vom Pfannenrand gelöst und bilden einen Narbenwulst, der sich retrahiert hat. \\
\hline Triple-Labrumläsion: & Labrum vom Glenoid und von Kapsel gelöst, zusätzlich Kapseltaschen durch Ablösung vom Skapulahals. \\
\hline Non-Bankart-Befund: & $\begin{array}{l}\text { Das inferiore glenohumerale Ligament (IGHL) setzt nicht wie normaler Weise am Labrum an, sondern am vorderen } \\
\text { Skapulahals. Es liegt deshalb eine mehr oder weniger weite vordere Kapseltasche vor. Dieser Befund liegt oft bei } \\
\text { atraumatischen Luxationsformen vor. }\end{array}$ \\
\hline $\begin{array}{l}\text { Substanzdefekt des } \\
\text { IGHL: }\end{array}$ & Narbiger Defekt; Elongationen im Bereich des unteren glenohumeralen Ligamentes, die zu einer Kapseltasche führen. \\
\hline $\begin{array}{l}\text { Quattro-Labrumläsi- } \\
\text { on: }\end{array}$ & Labrum vom Glenoid und der Kapsel gelöst mit zusätzlicher vorderer Kapseltasche und Substanzdefekt des IGHL \\
\hline HAGL-Läsion: & $\begin{array}{l}\text { Das inferiore glenohumerale Ligament ist an seiner Insertion des Humeruskopfes eingerissen. Oft kombiniert } \\
\text { mit Einrissen in der Subskapularissehne. }\end{array}$ \\
\hline GLAD-Läsion: & $\begin{array}{l}\text { Knorpelschaden am vorderen Pfannenrand ohne wesentliche Ablösung des Labrums, oft Folge einer direkten } \\
\text { Traumatisierung }\end{array}$ \\
\hline SLAP-Läsion: & Superiore Labrumläsion von anterior bis posterior unter Einbeziehung des Bizepssehnenankers \\
\hline
\end{tabular}

Tab. 3 Formen der Instabilitä nach C. Gerber [24]

- chronische Luxation

- unidirektionale Instabilität ohne Hyperlaxität

- unidirektionale Instabilität mit multidirektionaler Hyperlaxität

- multidirektionale Instabilität mit multidirektionaler Hyperlaxität

- multidirektionale Instabilität ohne Hyperlaxität

- willkürliche Instabilität - uni- oder multidirektional

\section{Aufklärung des Patienten}

$\mathrm{Zu}$ den speziellen operationsassoziierten Problemen gehört der Hinweis auf eine mögliche Rezidivluxation in $0 \%$ bis $\mathrm{zu}$ $30 \%$ (abhängig von der Technik), der Hinweis auf die Gelenkinfektion (und deren Prognose), Nervenverletzungen durch Dehnung, Stromanwendungen oder Instrumentenmanipulation. $\mathrm{Zu}$ den implantatspezifischen Nebenwirkungen können deren Dislokation oder auch eine lokale Synovialitis bei biodegradierbaren Materialien zählen. An postoperativen Störungen sind die z.T. gewollte als auch nicht gewollte Bewegungseinschränkung (Arthrofibrose bes. bei arthroskopischen Eingriffen innerhalb der ersten 3 Wochen nach der Erstluxation [12] zu erwähnen. Das wird für schulteraktive Sportler und Arbeiter von hoher Bedeutung sein.

\section{Narkose}

Zumeist wird für die Schulterstabilisation die sog. „Vollnarkose“ durchgeführt. Zusätzliche Nervenleitungsblockaden oder die intraoperative Injektion eines Lokalanästhetikums bzw. Morphinpräparates dienen der Schmerztherapie.

\section{Lagerung und Abdeckung}

Sowohl für die rein arthroskopischen als auch für die offenen Techniken lagern wir den Patienten halb sitzend, in der sog. „beach chair“-Position. Der Kopf und Rumpf sollten vor der Desinfektion schon ausreichend fixiert sein. Schulter und Arm werden zusammen abgewaschen und abgedeckt. Dafür sollte eine Person die Hand hoch halten, zwei steril Angezogene können von der Achsel aus ein UTuch kleben, das Schulterdach sowie die Halspartie wird mit einem geraden Tuch verdeckt.

\section{Spezielle offene Verfahrenstechniken}

Bankart beschrieb schon in den 30er Jahren des letzten Jahrhunderts die Kapsel-Labrumrefixation am vorderen Pfannenrand.

Sein Name steht heute noch für das gebräuchlichste und zugleich ANATOMISCHE OPERATIONSVERFAHREN, dem Bankartrepair.

Der Eingriff wurde bis in die 90er Jahre offen, d.h. mit einem vorderen Schnitt im Sulcus deltoideo-pectoralis oder inferior durch die Achsel durchgeführt. Unter der clavi-pectoralen Faszie erfolgte die Absetzung des Subskapularismuskels etwa $1 \mathrm{~cm}$ vor dem Tuberculum minus von der Schultergelenkskapsel. Die letztere wurde abschließend T-förmig entweder nahe dem kleinen Tuberculum oder nahe des Pfannenrandes eröffnet. Ziel des Eingriffes war es, die oftmals kaudal retrahierte vordere Kapsel mit dem Labrum zu mobilisieren und durch transossäre Fäden weiter kranial zu refixieren. Anzustreben ist eine Elevation der unteren Kapselanteile aus der 6- und 5-UhrPosition mit Anheftung weiter kranial, 


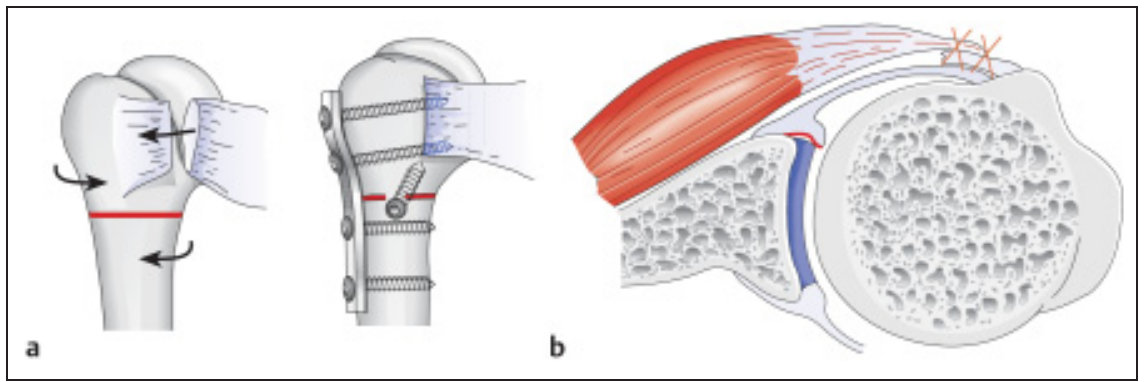

Abb.3au.b a Rotationsosteotomie n. Weber subkapital plus Putti-Platt-OP, 4,5-mm-Plattenosteosynthese; b der Labrumschaden bleibt hierbei unberührt.

z.B. bei $4.30 \mathrm{Uhr}$, an dem angefrischten Pfannenrand. Hierdurch wird die Kapsel im luxationsgefährdeten Bereich gespannt und durch eine Überlappung der unteren mit den oberen Anteilen (Kapselshift) verstärkt. Der Operationserfolg, gemessen an der Stabilität, beträgt für dieses Verfahren in der Literatur zwischen 92 und 100\% [22,23]. Die Entwicklung von Pfadenankern, Ankerdübeln und Staples sollte das Handling in der engen „Tiefe“ am vorderen Pfannenrand vereinfachen. Diese Implantate bilden einen Grundbaustein heutiger arthroskopischer Techniken.

Konkurrierend wurden im deutschsprachigen Raum jahrzehntelang EXTRAARTIKULÄRE OPERATIONSVERFAHREN zur primären Stabilisation von ventralen Schulterinstabilitäten durchgeführt. Zu den bekannten Verfahren zählt die Rotationsosteotomie nach Weber (Abb. 3). Hier erfolgt unterhalb des Collum chirurgicums eine horizontale Humerusosteotomie. Anschließend wird der distale $\mathrm{Hu}-$ merus, mit dem angewinkelten Unterarm als Zeiger, zwischen 20 und 30 Grad nach außen gedreht und der Oberarm z. B. mit einer 4,5-mm-Platte stabilisiert. Ziel der Operation ist es, die dorso-kraniale Kopfimpression (Hill-Sachs-Defekt) nach lateral-dorsal zu drehen, so dass dieser bei einer Außenrotation sich nicht mehr in den vorderen Pfannenrand einhaken kann. Zusätzlich ändert sich die (hintere und kaudale) Kapselspannung, was sich positiv auf die Reluxationshäufigkeit auswirken soll. Die Verkürzung, bzw. Dopplung der Subskapularissehne nach PuttiPlatt (Abb. 3) soll ventral die Kapselspannung beeinflussen und durch Einschränkung der Außenrotation Rezidivluxation verhindern. Kombiniert mit einer Weber-Operation wird die Bewegungseinschränkung in der Schulter bei akzeptabler Erfolgsrate wieder kompensiert [22].
Weniger populäre Verfahren in der deutschsprachigen Literatur sind u.a. die Versetzung der Korakoidspitze an den ventralen Skapulahals nach Bristow oder Latarjet (bei Revisionen ist der N. axillaris im Narbengewege besonders gefährdet), sowie der freie Knochenblocktransfer nach Eden-Hybbinette (Knochenblock wird auf den Skapulahals aufgeschraubt und soll hier Defekte füllen bzw. den vorderen Pfannenrand vergrößern), weil nach wenigen Jahren schon eine Arthrose auftritt. Dagegen empfiehlt Resch die Aufrichtung und Erweiterung der Glenoidebene mit einem J-förmigen Beckenkammspan [6] bei ventralen Pfannendefekten oder fehlgeschlagenen Rekonstruktionsoperationen (Abb.4).

\section{Spezielle arthroskopische Techniken}

Anhand der Fixationsverfahren lassen sich drei unterschiedliche arthroskopische Stabilisationstechniken unterscheiden (Tab.4). Die minimal invasiv kontrollierte Limbusfixation kann sowohl in der Seitenlage mit extendiertem Arm als auch halb sitzend („beach chair" Position) durchgeführt werden. Die letztere hat
Tab. 4 Gebräuchliche arthroskopische Verfahren zum Bankartrepair

1. Technik nach Morgan und Caspari

2. Technik mit Staples oder Schrauben

3. Technik mit Fadenanker



Abb.4 Defektaufrichtung des vorderen Pfannenrandes mit J-Span n. Resch.

den Vorteil, dass eine Konversion zur offenen Operation möglich ist (sofern die Weichteilschwellung dies noch erlaubt). Entscheidend ist das technische Equipment. Außer der Standard-30-Grad-Optik gehören dazu eine Rollenpumpe, voluminöse Trokare, kanülierte Nadeln, Raspeln, Fadenfänger (evtl. Shuttle-Relay $\left.{ }^{\mathrm{T}}\right)$, Kugelfraser oder spezielle Kapselfasszangen, sowie Anker- und Führungsinstrumente (Abb. 5). Mit dem (Einmal-)Instrumentarium ist der Operateur zumeist auf eine Fixationstechnik, z.T. auch an eine Zulieferfirma gebunden. Technische Erweiterungen oder Erneuerungen der Industrie können sich bei dem heutigen Kostendruck nur wenige „Spezialisten“ ohne Zeitverzögerung leisten.

Allen arthroskopisch assistierten Limbusverfahren gemeinsam sind die ersten

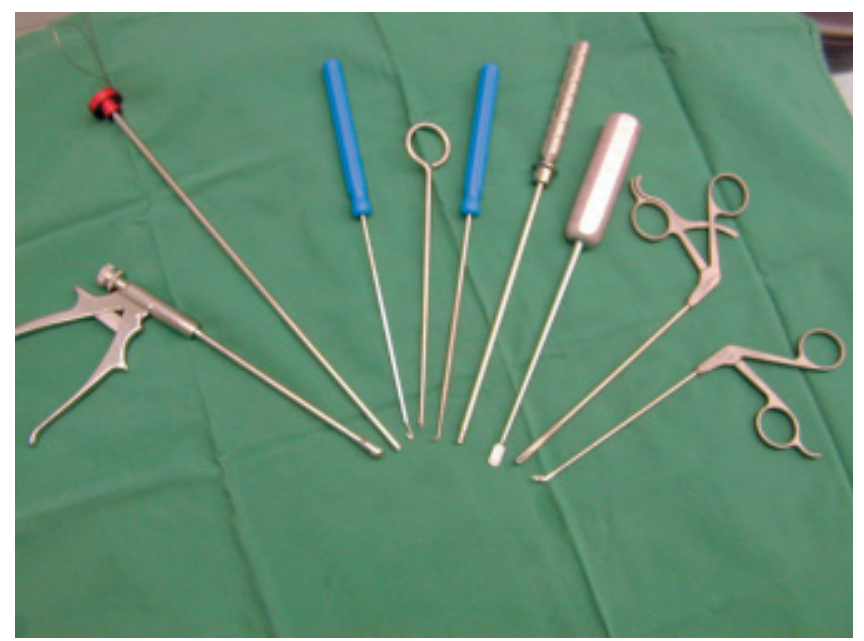

Abb. 5 Instrumentarium für arthroskopischen BankartEingriff von li nach rechts: Kapselfasszange, doppelläufiger Knotenschieber, Fadenhäkchen, Single-Knoten- und Fadenschieber, Tasthaken, Fadenschneider Raspel, Fadenfasszange, „bird-pick“Zange. 
Tab.5 Metaanalyse der Literatur zum Stabilisationserfolg anteriorer Luxationen mit verschiedenen Techniken:

\begin{tabular}{|c|c|c|c|c|c|}
\hline Studie & Technik & $\begin{array}{l}\text { Pati- } \\
\text { enten } \\
\mathrm{N}\end{array}$ & Indikation & FU (Mon.) & Rez. (\%) \\
\hline Rowe $1978^{\dagger, 23}$ & offene Bankart-Naht & 145 & traumatische Instabilität & 72 & 3,5 \\
\hline $\begin{array}{l}\text { Morgan } \\
1987^{*}\end{array}$ & transglenoid. Naht & 25 & vordere traumatische Instabilität & 17 & 0 \\
\hline Wolf $1988^{*}$ & $\begin{array}{l}\text { Schraube und Unter- } \\
\text { legscheibe }\end{array}$ & 23 & Bankart-Läsion & $5-26$ & 0 \\
\hline $\begin{array}{l}\text { Matthews } \\
1988^{*}\end{array}$ & Staple Kapsulorhaphie & 25 & $\begin{array}{l}12 \text { rez. vord. Instabilität } \\
12 \text { rez. vord. Subluxationen }\end{array}$ & 36 & 12 \\
\hline $\begin{array}{l}\text { Hawkins } \\
\text { 1989* }\end{array}$ & Staple capsulorrhaphy & 50 & $\begin{array}{l}15 \text { chron. Schulterluxationen } \\
35 \text { chron. Schultersublux. }\end{array}$ & 39 & 16 \\
\hline Caspari $1991^{*}$ & transglenoid. Naht & 49 & traumatische vordere Instabilität & $24-72$ & $?$ \\
\hline $\begin{array}{l}\text { Detrisac } \\
1991^{*}\end{array}$ & Staple Kapsulorhaphie & 148 & traumatische Instabilität & 48 & 11 \\
\hline $\begin{array}{l}\text { Benedetto } \\
1992^{*}\end{array}$ & transglenoid. Naht & 22 & $\begin{array}{l}14 \text { vord. Erstluxationen } \\
8 \text { rez. vord. Subluxationen }\end{array}$ & 29 & 0 \\
\hline $\begin{array}{l}\text { Landsiedl } \\
\text { 1992* }\end{array}$ & transglenoid. Naht & 65 & rezidivierende vord. unidirektionale Instabilität & 35 & 13,8 \\
\hline $\begin{array}{l}\text { Coughlin } \\
1992^{*}\end{array}$ & Staple Kapsulorhaphie & 47 & $\begin{array}{l}34 \text { traumatische Instabilität } \\
13 \text { Subluxationen }\end{array}$ & 48 & 25 \\
\hline Lane $1993^{*}$ & Staple Kapsulorhaphie & 54 & $\begin{array}{l}83 \% \text { mindestens } 1 \text { Luxation } \\
17 \% \text { rez. Subluxationen }\end{array}$ & 39 & 33 \\
\hline Grana 1993* & transglenoid. Naht & 27 & $\begin{array}{l}4 \text { Pat. } x \text { akute Instabilität } \\
23 \text { Pat. } x \text { chron. oder rez. Instab. }\end{array}$ & 36 & 44 \\
\hline Walch $1995^{26}$ & transglenoid. Naht & 59 & rezidivierende vordere Luxation & 49 & 49 \\
\hline $\begin{array}{l}\text { Hayashida } \\
1998^{7}\end{array}$ & transglenoid. Naht & 82 & traumatische Instabilität & 40 & 16 \\
\hline $\begin{array}{l}\text { Boszotta } \\
1999^{1}\end{array}$ & Transglenoid. Naht & 67 & traumat. Erstluxationen, Sportler, < 40 J. & 67 & 6,9 \\
\hline $\begin{array}{l}\text { Kandziora } \\
2000^{13}\end{array}$ & $\begin{array}{l}\text { FASTak }^{\mathrm{TM}} \\
\text { transglenoid. Naht }\end{array}$ & $\begin{array}{l}55 \\
108\end{array}$ & traumat. Instabilität & $\begin{array}{l}38 \\
54\end{array}$ & $\begin{array}{l}32,4 \\
16,4\end{array}$ \\
\hline Lyons $2001^{17}$ & Laser-Kapsulorhaphie & 27 & multidirektionale Instabilität & 27 & 3,7 \\
\hline Fealy $2001^{3}$ & Suretac & 52 & $\begin{array}{l}49 \times \text { traumat. Instabilität } \\
3 \times \text { atraumatische Luxation }\end{array}$ & 42 & 21 \\
\hline Mishra $2001^{20}$ & $\begin{array}{l}\text { Suretack }{ }^{\mathrm{TM}} \text { oder Mi- } \\
\text { tek }^{\mathrm{TM}}+\text { jeweils elektro- } \\
\text { thermale Kapsulo- } \\
\text { rhaphie }\end{array}$ & 42 & traumatische vordere Instabilität & 28 & 7 \\
\hline $\begin{array}{l}\text { Summe/ } \\
\text { Mittelwert }\end{array}$ & & 1172 & & 140,4 & $\mid 16,4$ \\
\hline
\end{tabular}

* zit. aus Walch, 1995

$\dagger$ einzige Studie in der Metaanalyse mit offener Operationstechnik

$\mathrm{FU}=$ Nachuntersuchungszeitraum; Rez. $=$ Rezidivrate

Schritte. Dazu gehört der Standardzugang von dorsal („soft spot“ zwischen M. infraspinatus und M. teres minor, Abb.6) mit dem orientierenden Blick auf die vordere Kapsel sowie auf den Limbusrand. Begleitverletzungen erfasst der Operateur durch eine erste Rund-um-Inspektion.
Anschließend wird mit einer langen Kanüle die Position für den vorderen $\mathrm{Zu}$ gang bestimmt, der in den meisten Fällen etwa 1 bis $1,5 \mathrm{~cm}$ kaudal des Proc. coracoideus liegt (Abb.6), um hierdurch die unteren Kapsel-Limbusanteile zu erreichen. Der Instrumentenzugang wird mit einem großen Trokar (7,0 - 8,4 mm) eröffnet. Ein dritter Zugang für Instrumente oder Optik kann etwa $1 \mathrm{~cm}$ kranial des Proc. coracoideus als vorderer superiorer Port gelegt werden. Mit der Raspel (Abb.7) wird zunächst das retrahierte Kapsel-Limbusgewebe am vorderen und 




Abb.6 Zugänge für den arthroskopischen Bankart-Repair; dorsalksoft spot; ventral superior, ventral parakorakoidal, ventral inferior.

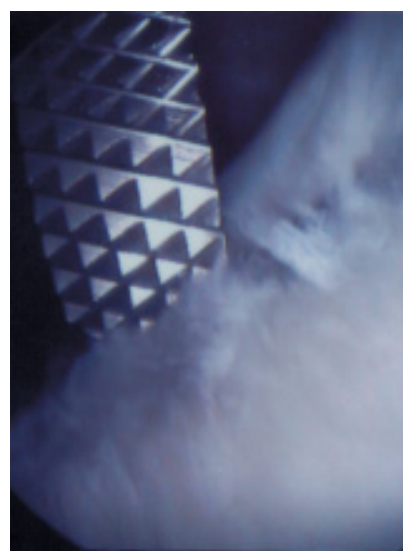

Abb. 7 Raspel für die arthroskopisch kontrollierte Anfrischung des vorderen Pfannenrandes.

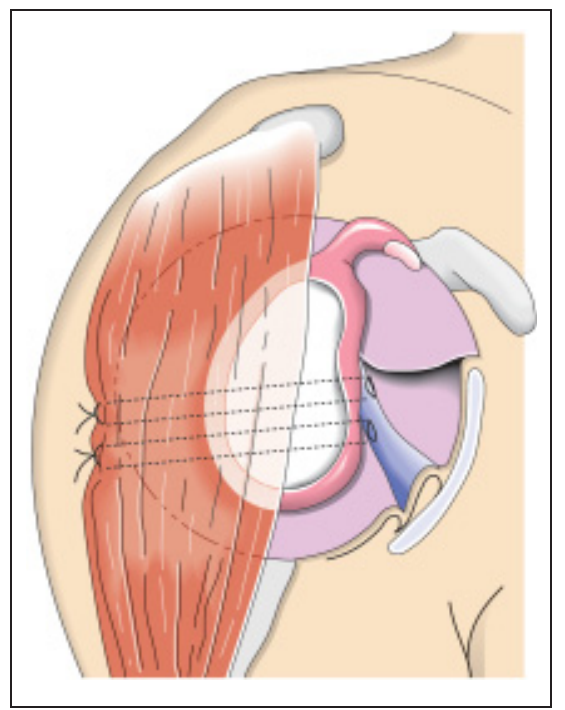

Abb. 8 Transglenoidale Nahttechnik nach Caspari.

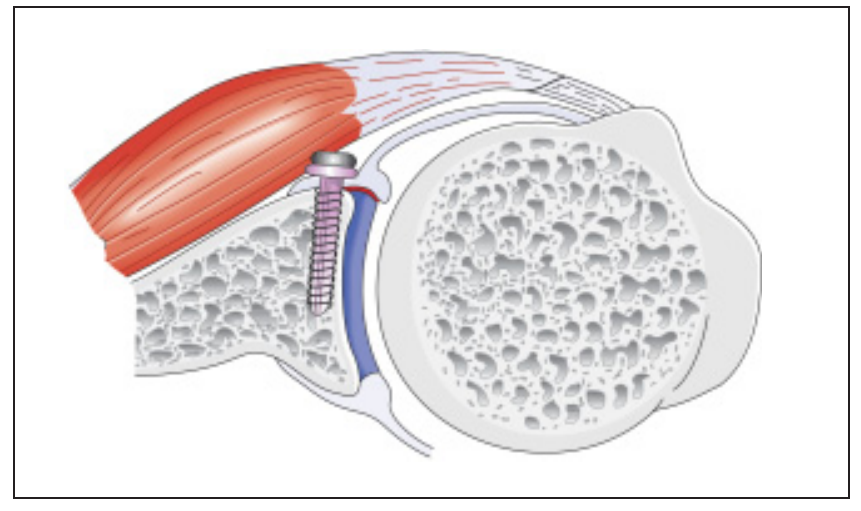

Abb. 9 Extraartikuläre Schraubenfixation einer BankartLäsion.

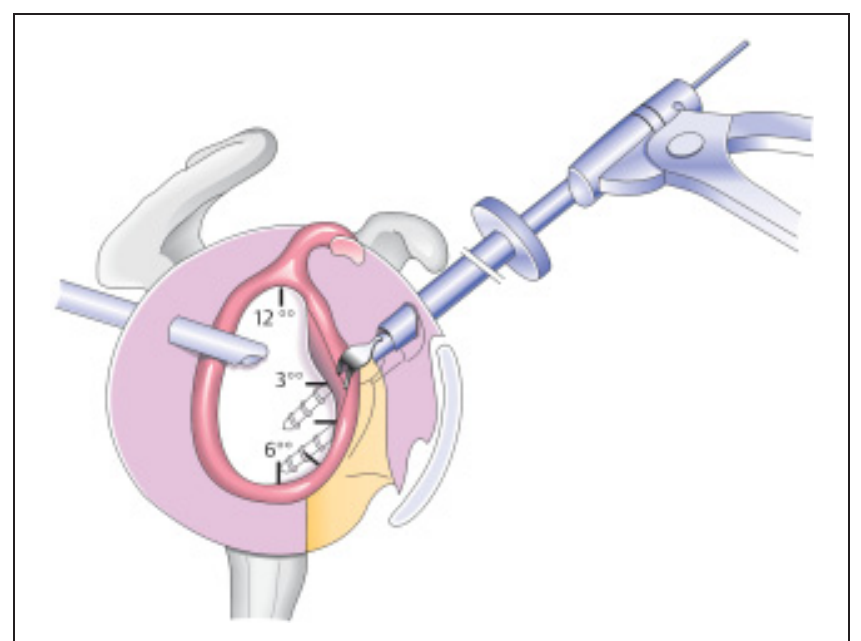

Abb.10 Einbringen eines 2-Faden-Ankers bei 3 Uhr. Mit der Kapselfasszange wurde zuvor mobilisiertes Gewebe gefasst und nach kranial gezogen.

unteren Pfannenrand von ca. 2 bis ca. 6 Uhr mobilisiert und anschließend der Insertionsbereich angefrischt (z.B. mit der Kugelfräse). Die weiteren Schritte beginnen am unteren Limbus-Kapselkomplex und sind auf die Fixationstechnik abgestimmt.

Nach Morgan und Caspari ist die transglenoidale Nahttechnik benannt (Abb.8). Dazu wird z.B. mit einer doppelläufigen Kanüle oder einer kanülierten Fasszange Kapsel- und Limbusgewege kaudal gefasst und weiter kranial gegen den vorderen Pfannenrand gehalten. Ein K-Draht mit Öse spießt das gefasste Gewebe auf, er wird nach dorsal durchgebohrt, bis er extrakutan wieder zu fassen ist. Ein zweiter K-Draht verläuft parallel dazu, so dass beide Ösen mit je einem Fadenende armiert werden können. Beim Spannen auf der Rückseite entsteht somit ventral eine U-förmige Limbusfixation. Der Knoten liegt dann auf der Faszie der dorsalen Rotatoren. Mindestens zwei dieser U-Nähte sind für die Anheftung des abgelösten Limbus erforderlich.
Resch favorisiert die extraartikulären Schrauben - (2,7 mm Titan, Abb.9) oder biodegradable Suretak ${ }^{\mathrm{TM}}$-Fixation im Pfannenrandbereich für eine stabile Limbusanheftung [21]. Lagerung und Zugänge unterscheiden sich von dem obigen Verfahren nicht wesentlich. Mit einer zförmigen Bewegung führt der Operateur die Führungskanüle durch den Subscapularismuskel an die Kapsel. Nach der Limbusmobilisation und Pfannenanfrischung wird jedoch der Zieldraht zur Kapselanheftung im Gelenk nicht sichtbar. Den Draht und die anschließende Schraube bzw. Suretack ${ }^{\mathrm{TM}}$ ohne visuelle Gegenkontrolle korrekt in den schräg verlaufenden Pfannenhals und nicht an diesem vorbei gleiten zu lassen, erfordert Übung.

Die häufigste Anwendung findet heute jedoch die intraartikuläre Fadenankernaht (Abb.10,11) z.B. mit einem FASTak ${ }^{\mathrm{TM}}$ oder Mitek ${ }^{\circledR}$-Anker [2]. Unabhängig vom verwendeten Ankertyp können 3 Nahtvariationen unterschieden werden: (A) Am einfachsten ist es, das mobilisierte Limbus- und Kapselgewebe mit einer Zange zunächst kaudal $\mathrm{zu}$ fassen, mit 
Tab. 6 Technische Fehler der arthroskopischen Bankart-Operation:

1. unzureichende Anfrischung des unteren Glenoidrandes zwischen 4 und 6 Uhr

2. unzureichende Mobilisation der Kapsel mit zu wenig medialem sowie superiorem Shift des unteren Labrum-Kapselkomplexes einschließlich des Lig. glenohumerale inferius

3. zu mediale Platzierung des Fixationsimplantates in Bezug zum Glenoidrand

4. zu wenig Labrum-Kapselgewebe gefasst

5. unzureichender Andruck des gefassten Labrum-Kapselgewebes an den Glenoidrand dem Anker mittig zu perforieren und den Anker weiter kranial am Gelenkrand einzuschrauben. Mit zusätzlichen Ankerfäden kann zwischen den Ankern eine Uförmige Naht geknüpft werden. Diese eher flächenförmige Anheftung erlaubt jedoch noch relativ viel Kapselbewegungen gegenüber dem Glenoid und ist damit nicht sehr vorteilhaft. (B) In der zweiten Variation wird wiederum mit der Fasszange Limbus- und Kapselgewebe gefasst, mit einem Anker durchspießt, der wiederum in den Pfannenrand eingeschraubt wird. Nun lupft der Operateur eines der Ankerfäden am Limbusunterrand mit einem Tast- oder Fadenhäkchen gelenkseitig hervor und zieht diesen aus der vorderen Instrumentenkanüle erneut heraus. Der Knoten fixiert Limbus- und Kapselgewebe direkt (Abb.11). (C) Technisch aufwändiger, aber effektiver, ist die Verwendung von sog. „SutureHooks $^{\circledR}$ ", die von Firmen in verschiedenen Winkeln, Durchmessern oder korkenartigen Formen angeboten werden. Zunächst wird ein Fadenanker über das ventrale Portal in der 4.30-Uhr-Position in den vorderen Pfannenrand eingeschraubt und eines der beiden Fadenenden durch ein drittes Portal ausgeleitet. Dann wird mit einem geeigneten „Suture-Hook" von ventral Kapsel- und Limbusgewebe aufgeladen. Mit einem Fangfaden (z.B. "Shuttle-Relay ${ }^{\mathrm{TM} \text { “ }}$ ) gelingt es den zuvor ausgeleiteten Faden in den Instrumententrokar wieder zurückzuziehen. Mit dem „Suture-Hook ${ }^{\circledR “}$ kann übersichtlich und gezielt Labrum-Kapselgewebe von kaudal nach kranial aufgeladen und mit dem abschließenden Knoten gespannt werden. Für die intraartikulären Fixationstechniken sind mindestens 2 Anker notwendig, 3 bis 4 Fixationsknoten geben jedoch einen besseren Halt. Additiv gewinnt die ventrale Kapselraffung mittels Thermo- oder Laserkoagulation [17] kombiniert mit einer Fadenraffung [20] zunehmend an Bedeutung (Tab.5).

\section{Tuberculum-majus-Fraktur}

Vordere Luxationen mit zusätzlicher Tuberculum-majus-Fraktur heilen zumeist mit einer Bewegungseinschränkung stabil aus.

Unter 90 Erstluxationen mit einer Tuberculum-majus-Fraktur fand McLaughlin nur eine einzige Rezidivluxation [19]. Dislokationen des Fragmentes von mehr als $2 \mathrm{~mm}$ nach kranial erhöhen das spätere Impingementrisiko und sollte gerade bei jüngeren Patienten großzügig reponiert und fixiert werden. Ein Tuberculumhochstand von $5 \mathrm{~mm}$ und mehr ist auch bei älteren Patienten zumeist mit einem schmerzhaften Impingement verbunden und sollte deshalb operativ angegangen werden.

Die „Luxatio erecta“ (mit mögl. Tuberculumfraktur) sollte ebenfalls operativ stabilisiert werden.

\section{Rotatorenmanschettenruptur}

Ab dem 40. Lebensjahr erhöht sich mit der Schulterluxation zunehmend das Risiko einer begleitenden Rotatorenmanschettenruptur auf 40 bis $60 \%$ [4].

Hieraus können sich Funktionseinschränkungen und versicherungsrelevante Ansprüche ergeben. Kann ein Patient wenige Tage nach der Reposition die Schulter nicht aktiv heben oder außenrotieren ist unbedingt diese Diagnose sonographisch oder kerspintomographisch abzuklären. Teilrupturen oder kleinere Supraspinatussehneneinrisse behandeln wir konservativ, komplette Sehnenabrisse refixieren wir transossär über einen Minisplitzugang des Deltamuskels. Erweiterungen der Intervallregion zwischen Supraspinatus- und Subskapularissehne werden durch Kapselnähte adaptiert.

Während im NMR vermutete Subskapularissehnenteil- oder komplette Rupturen sich arthroskopisch oftmals nicht bestätigten (besonders wenn die Bizepssehne

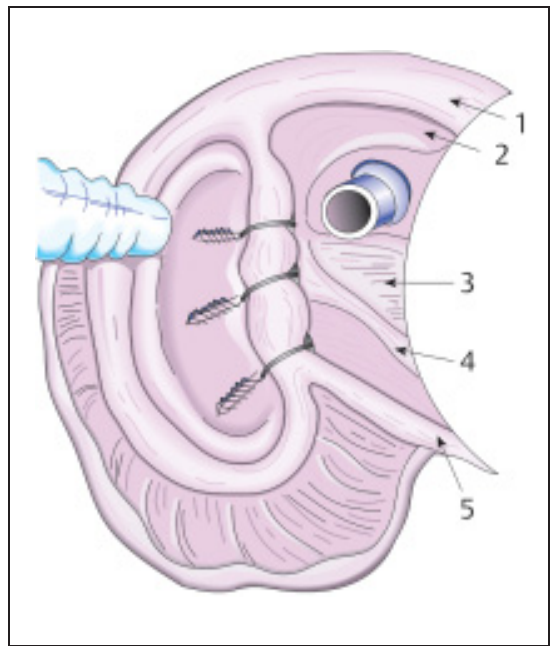

Abb.11 Anatomische Rekonstruktion eines Labrum-Kapselschadens mit 3 Fadenankern. 1 = lange Bizepssehne, 2 = Lig. glenohumerale superius, $3=$ Subskapularissehne, $4=$ Lig. glenohumerale medius, $5=$ Lig. glenohumerale inferius. Das ventrale Arbeitsportal sollte für das Einbringen des untersten Fadenankers tiefer liegen als in der Zeichnung.

nicht luxiert und die Supraspinatussehne intakt ist), macht diese Diagnose wegen der erschwerten Spätrekonstuktion eine frühzeitige sowie sorgfältige intraoperative Abklärung und Rekonstruktion erforderlich.

\section{Operative Techniken bei dorsaler Instabilität}

Zunächst muss in der Indikationsstellung zwischen den traumatischen und den atraumatischen sowie chronischen Luxationsformen differenziert werden. Dorsale Luxationen durch erhebliche Krafteinwirkung hinterlassen zumeist einen ordentlichen Defekt im ventralen Humeruskopf, z. T. auch mit Ein- oder Abriss der Subscapularissehne. Deshalb ist die CTDiagnostik zur Operationsplanung hilfreich. Hier empfiehlt sich die sofortige Operation, wenn keine geschlossene Reposition gelingt. Der Defekt kann entweder mit dem abgerissenen Tuberculum Minusfragment während der Refixation aufgefüllt werden oder eine Defektauffüllung mit Beckenkamm-, Fremdspongiosa oder der Subskapularissehne nach McLaughlin ist erforderlich $[9,16]$, wenn ein retrogrades Hochstößeln der imprimierten Kalotte nicht möglich ist. Die Lagerung des Armes erfolgt postoperativ auf einer Schiene in geringer Außenrotation. Bei den so behandelten dorsalen Luxationen sahen wir nach der Defektauffüllung im Verlauf keine Rezidive. 
Komplexer ist das Vorgehen nach atraumatischen oder chronisch verhakten hinteren Verrenkungen $[9,27]$. Die erweiterte und ausgedünnte Kapsel, eine Kapselinsuffizienz in der Intervallregion, neuromuskuläre Defizite und die Persönlichkeit des Patienten sollten in das Behandlungskonzept mit einbezogen werden.

\section{Nachbehandlung}

Die Nachbehandlung untergliedert sich nach anatomischen Rekonstruktionen am vorderen Pfannenrand der Schulter in zwei Phasen. Zunächst darf die Heilung der Kapselnaht durch postoperative Bewegungen nicht gefährdet werden. Hierzu legen wir einen Gilchristverband für 1-2 Wochen an, die Außenrotation sowie Ellvation über 90 Grad ist für insgesamt 6 Wochen limitiert. Aktive Bewegungen (z.B. PNF-Diagonale) und isometrische Muskelübungen zur Armkontrolle werden unter physiotherapeutischer Anleitung durchgeführt. Nach 6 Wochen geben wir das Bewegungsausmaß der Schulter frei. Bei Rotatorenmanschettenrupturen oder Frakturversorgungen wird der Belastungsaufbau modifiziert. Eine Armschiene in Außenrotation legen wir für 4 Wochen nach Rekonstruktion einer dorsalen Luxation an. Schulterabduktionsschienen haben darüber hinaus wegen des schlechten Tragekomforts an Bedeutung verloren.

\section{Diskussion}

Vorangetrieben durch die Entwicklung arthroskopischer und nicht invasiver bildgebender Verfahren sowie die Herstellung geeigneter Implantate mit kanülierten Instrumenten konnten Therapiekonzepte für die Behandlung der vorderen Schultergelenksverrenkungen vereinfacht und standardisiert werden. In einer Metaanalyse der Literatur zeigen sich jedoch in Bezug auf die angewendeten Techniken erhebliche Unterschiede der (Tab.5) Rezidivraten. Die arthroskopische Stabilisation ist anspruchsvoll, Versager sind bei einer unzureichenden Technik vorprogrammiert (Tab.6). Am zuverlässigsten ist bis heute nach wiederholten vorderen Verrenkungen bei unidirektionaler Instabilität der offene Kapseleingriff im Sinne eines Bankartrepair (Tab.5). Nur durch eine gezielte Patientenselektion, wie sie für die bisherigen offenen Verfahren kaum durchgeführt wurde, konnten wenige Autoren bei arthroskopischem Vorgehen annähernd vergleichbare Stabilitätsresultate erzie-

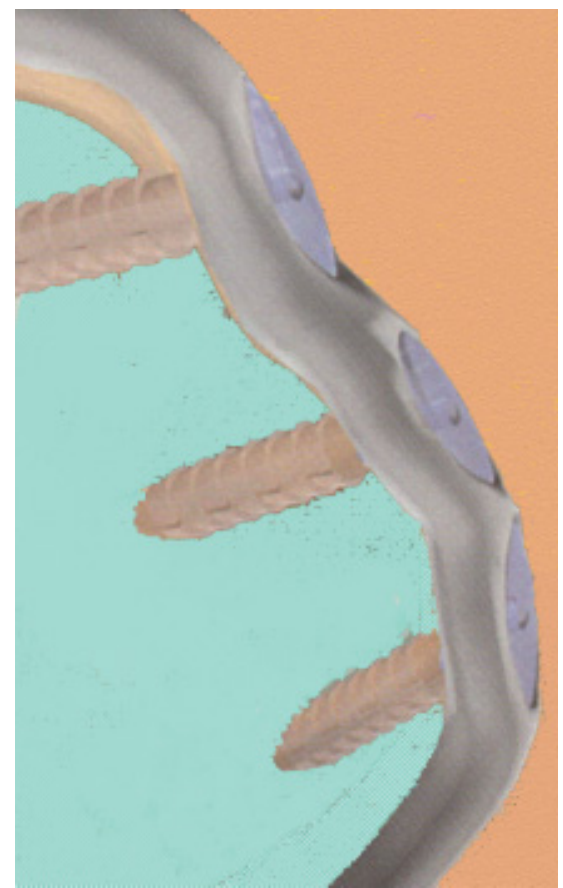

Abb.12 „Simple“ Labrumfixation in der Werbung.

len [20]. Dabei kommt es nicht nur auf die alleinige Limbusfixation an, wie dies z.B. in manchen Werbeprospekten der Industrie dargestellt wird (Abb.12), entscheidend ist die zusätzliche anatomische Reinsertion des Ligamentum glenohumerale inferius am vorderen Pfannenrand. Weiterhin ist die präoperative Erfassung einer zusätzlichen Hyperlaxität (positives Sulkus-Zeichen) für das Behandlungskonzept bedeutsam. Die minimal invasive Operationstechnik hat daher nicht das offene Vorgehen abgelöst, sondern sie stellt eine zusätzliche Möglichkeit zur differenzierten Behandlung von Schulterinstabilitäten dar. Die Ergebnisse machen ebenfalls deutlich, dass eine einzige oder zu simple Technik den Anforderungen eines sportlich ambitionierten Klientel nicht genügen. Auf der anderen Seite sollten wiederholte Rezidivluxationen nicht immer erst abgewartet werden. Wir befürworten deshalb eine individuelle Risikoanalyse im Gespräch mit dem Patienten und die frühzeitige arthroskopische Stabilisation nach der ersten traumatischen Luxation bei unter 30-Jährigen. Notfallindikationen sind für uns die verhakte oder nicht geschlossen reponierbare Verrenkung. Knöcherne Bankartfrakturen mit einer Gelenkstufe, dislozierte Tuberculum-majus-Frakturen (von mehr als $4 \mathrm{~mm}$ ) sowie frische Rotatorenmanschettenrupturen (insbesondere der Subskapularissehne) sollten opera- tiv frühzeitig angegangen werden. Die konservativ-funktionelle Therapie von Schulterverrenkungen hat nach Ausschluss dieser wichtigen Begleitverletzungen jedoch auch heute noch einen berechtigten Stellenwert.

\section{Literatur}

${ }^{1}$ Boszotta H, Helperstorfer W. Arthroskopische, transglenoidale Versorgung der traumatischen Erstluxation der Schulter. Arthroskopie 1999; $12: 177-182$

${ }^{2}$ Cole BJ, Romeo AA. Arthroscopic shoulder stabilization with suture anchors: Technique, technology, and pitfalls. Clin Orthop 2001; 390: $17-30$

${ }^{3}$ Fealy St, Drakos MC, Allen AA, Warren RF. Arthroscopic Bankart repair. Clin Orthop 2001; 390: $31-41$

${ }^{4}$ Gerber CH. Angeforderter Kommentar. Orthopäde 1989; 18: $255-256$

${ }^{5}$ Habermeyer P, Jung D, Ebert T. Behandlungsstrategie bei der traumatischen vorderen Erstluxation der Schulter. Unfallchirurg 1998; 101: $328-341$

${ }^{6}$ Habermeyer P. Schulterchirurgie. Urban \& Fischer Verlag 2002; 3. Aufl.

${ }^{7}$ Hayashida K, Yoneda M, Nakagawa Sh, Okamura K, Fukushima S. Arthroscopic Bankart suture repair for traumatic anterior shoulder instability: analysis of the causes of a recurrence. Arthroscopy 1998; 14: 295-301

${ }^{8}$ Hedtmann A, Heers G, Heidersdorf S. Bildgebende Verfahren an der Schulter. Arthroskopie 2001; 14: 74-93

${ }^{9}$ Heller KD, Forst J, Forst R. Differentialtherapie der traumatisch induzierten persistierenden hinteren Schulterluxation. Unfallchirurg 1995; 98: 6-12

${ }^{10}$ Hintermann B, Gächter A. Arthroscopic findings after shoulder dislocation. Am J Sports Med 1995; 23: 545 - 551

${ }^{11}$ Hovelius L. Anterior Dislocation of the shoulder in teen-agers and young adults: five year prognosis. J Bone Joint Surg 1987; 69-A: 393 - 399

12 Jung D, Habermeyer P, Lehmann L. Arthrofibrose bei arthroskopischer Stabilisierung der primären Schulterluxation. Arthroskopie 2000; 13: $237-240$

13 Kandziora F, Jäger A, Bischof F, Herresthal J, Starker M, Mittlmeier Th. Arthroscopic labrum refixation for post-tramatic anterior shoulder instability: suture anchor versus transglenoid fixation technique. Arthroscopy 2000; 16: 359-366

14 Koch M, Kienle P, Titz Th, Meeder PJ. Die subacromiale Schulterluxation. Chirurg 1999; 70: $1361-1363$

${ }^{15}$ Kroner K, Lind T, Jensen J. The epidemiology of shoulder dislocations. Arch Oprthop Trauma Surg 1989; 108: 288-290

${ }^{16}$ Letts RM, Kawam M, Davidson D. Dorsaler Knochenblock und Kapselplastik zur Behandlung der hinteren Instabilität der Schulter bei Jugendlichen. Operative Orthopädie und Traumatologie 2000; 12: 87-94

17 Lyons TR, Griffith PL, SavoieIII FH, Field LD. Laser-assisted capsulorrhaphy for multidirectional instability of the shoulder. Arthroscopy 2001; 17: 25 - 30

${ }^{18}$ Matsen FA, Thomas SC, Rockwood CA. Glenohumeral instability. In Rockwood CA, Matsen FA [Hrsg.]. The Shoulder. Saunders, Philadelphia 1990 
${ }^{19}$ Mc Laughlin HL, Mc Lellan DI. Recurrent anterior dislocation of the shoulder II. A comparative study. J Trauma 1967; 7: 191-201

${ }^{20}$ Mishra DK, Fanton GS. Two-Year outcome of arthroscopic bankart repair and electrothermal-assisted capsulorrhaphy for recurrent traumatic anterior shoulder instability. Arthroscopy 2001; 17: 844-849

${ }^{21}$ Resch H, Beck E. Arthroskopie der Schulter. Springer Verlag Wien, New York 1991

${ }^{22}$ Richter J, Lacher B, Stratmann B, Ekkernkamp A, Muhr G. Sport und Berufsfähigkeit nach Stabilisation rezidivierender Schultergelenksluxationen. Unfallchirurg 1997; 100: $198-204$

${ }^{23}$ Rowe CR, Patel D, Southmayd WW. The Bankart Procedure. J Bone Joint Surg 1978; 60-A: 1 - 16
${ }^{24}$ Schneeberger AG, Hersche O, Gerber C. Die instabile Schulter. Unfallchirurg 1998; 101: $226-231$

${ }^{25}$ Spatschil A, Landsiedl F, Anderl W, Imhoff A, Seiler H, Vassilev I, Klein W, Boszotta $\mathrm{H}$, Hoffmann F, Rupp St. AGA-Schulterluxationsstudie. Arthroskopie 2000; 13: 249-252

${ }^{26}$ Walch G, Boileau P, Levigne Ch, Mandrino A Neyret Ph, Donell S. Arthroscopic stabilization for recurrent anterior shoulder dislocation: results of 59 cases. Arthroscopy 1995; 11: $173-179$

${ }^{27}$ Wolf EM, Eakin CL. Arthroscopic capsular plication for posterior shoulder instability. Arthroscopy 1998; 14: 153-163
Dr. med. Jens Richter Oberarzt

Prof. Dr. med. Andreas Dávid Klinikdirektor

Klinik für Unfall- und Wiederherstellungschirurgie Klinikum Wuppertal-Barmen Heusnerstr. 40

D-42283 Wuppertal 